\title{
Structure of Acidic Polysaccharide Produced by Strain No. 626 of Aeromonas hydrophila
}

\author{
Kouichi Ito, Hiroshi Yoshino and Seinosuke UEDA \\ Department of Food Science and Technology, Faculty of Agriculture, \\ Kyushu University, Fukuoka 812, Japan \\ Received January 6, 1982
}

The structure of acidic polysaccharide from strain No. 626 of Aeromonas hydrophila was studied. The possible structure shown was proposed from the results of methylation analysis, acetolysis, partial acid hydrolysis and Smith degradation.

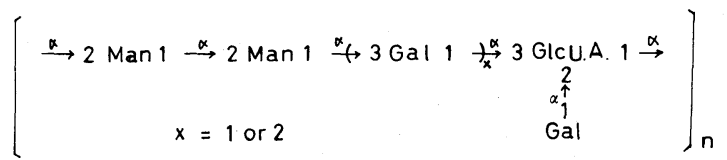

Usually organisms such as Serratia, ${ }^{1)}$ Klebsiella, $^{2)}$ Alcaligenes $^{3)}$ and Azotobacter $^{4)}$ produce same extracellular polysaccharide regardless of the carbon source used. In our previous paper, ${ }^{5)}$ we reported that strain No 626 of Aeromonas hydrophila isolated in our laboratory produced levan and a slimy acidic polysaccharide from sucrose and raffinose, and mainly a slimy acidic polysaccharide from monosaccharides and disaccharides, other than sucrose. In this paper, we describe the structure of the acidic polysaccharide produced by strain No. 626 of Aeromonas hydrophila.

\section{MATERIALS AND METHODS}

Production and purification of acidic polysaccharide. Production and purification of acidic polysaccharide were performed as described previously. ${ }^{5}$ )

Analytical methods. Paper chromatography was usually performed on Toyo roshi No. 51 A paper by the ascending method with the following solvent system $(\mathrm{v} / \mathrm{v}) ; n$ butanol-pyridine--water $(6: 4: 3)$. Reducing sugars on the paper were detected by spraying alkaline silver nitrate. ${ }^{6)}$ Gas-liquid chromatography (GLC) was carried out with a Nihondenshi gas-liquid chromatograph Model JGC-20 KFP fitted with a flame ionization detector using a glass column $(0.4 \times 200 \mathrm{~cm})$ packed with $3 \%$ ECNSS-M on Gas Chrom Q. Neutral sugars, sugar alcohols and methylated sugars were converted into the corresponding alditol acetate derivatives $^{7)}$ before separation by GLC. Total carbohydrates were generally determined by the phenolsulfuric acid $\operatorname{method}^{8)}$ and reducing sugars by the Somogyi-Nelson method..$^{9)}$ The optical specific rotation of a $0.5 \%$ polysaccharide solution in water was measured with a Unicon Automatic Digital Polarometer PM-101.

Reduction of acidic polysaccharide and oligosaccharide. Carboxyl groups in the acidic polysaccharide and oligosaccharide were reduced by the method of Dudman. ${ }^{10}$ ) This is a modification of the Taylor and Conrad technique. ${ }^{11)}$ The polysaccharide $(30 \mathrm{mg})$ was dissolved in water and buffered with 2-( $N$-morpholino)-ethanesulfonic acid $(1.25 \mathrm{M}, \mathrm{pH} 4.75,1 \mathrm{ml})$; sodium hydrogen-carbonate ( $5 \%$ solution) was added dropwise to adjust the $\mathrm{pH}$ of the solution to 4.75 and 1-ethyl-3-(3-diethylaminopropyl) carbodiimide (EDC) $(190 \mathrm{mg})$ was added. The $\mathrm{pH}$ of the solution was maintained at 4.75 by addition of hydrochloric acid $(0.1 \mathrm{M})$ and more EDC $(50 \mathrm{mg})$ was added $1 \mathrm{hr}$ later. After a further $1 \mathrm{hr}$, sodium borohydride $(1.9 \mathrm{~g}$ in $25 \mathrm{ml}$ of water) was added dropwise with stirring for $1 \mathrm{hr}$, during which time hydrochloric acid (5.0 M) was added to maintain the solution at $\mathrm{pH}$ 8.0. The solution was kept overnight to allow evolution of hydrogen to cease, which was then dialyzed exhaustively, deionized with Dowex $50\left(\mathrm{H}^{+}\right.$form $)$and lyophilized. In the case of acidic oligosaccharide, after keeping the solution overnight to allow evolution of hydrogen to cease, the solution was passed through Dowex $50\left(\mathrm{H}^{+}\right.$form $)$and evaporated to dryness. Methanol was added to it and the mixture was evaporated to remove boric acid as methyl ester. After that, impurities were removed by gel filtration (Bio-gel P2 ), and the reduced oligosaccharide was collected. 
Methylation. Dry samples of polysaccharide or oligosaccharide $(1 \sim 20 \mathrm{mg})$ were dissolved in dehydrated dimethyl sulfoxide $(2 \mathrm{ml})$ and treated for $3 \mathrm{hr}$ at room temperature with methylsulfinyl carbanion ${ }^{12)}(0.5 \mathrm{ml})$, and the saccharide alcoxide, so formed, was treated with methyl iodide $(1.5 \mathrm{ml})$ at $20^{\circ} \mathrm{C}$ according to the method of Hakomori. ${ }^{13)}$ The methylated polysaccharide or oligosaccharide was hydrolyzed by heating in a sealed tube with $0.5 \mathrm{ml}$ of $90 \%$ formic acid for $7 \mathrm{hr}$ at $100^{\circ} \mathrm{C}$, and then further with $0.5 \mathrm{ml}$ of $2 \mathrm{~N}$ trifluoroacetic acid for $6 \mathrm{hr}$. The acids were removed by repeated evaporations with water. The hydrolyzates were converted into the corresponding alditol acetates. The products were analyzed by GLC on a column with $3 \%$ ECNSS-M on Gas Chrom Q.

Acetolysis of polysaccharide. One gram of polysaccharide was dissolved in a mixture of $10 \mathrm{ml}$ of acetic anhydride and $1.2 \mathrm{ml}$ of conc. sulfuric acid, and the mixture was allowed to react for $3.5 \mathrm{hr}$ at $60^{\circ} \mathrm{C}$. The resulting solution was poured into cold sodium acetate solution and neutralized with sodium carbonate. The acetylated solution was extracted with $20 \mathrm{ml}$ of chloroform (3 times). The chloroform layer was washed with saturated sodium chloride solution and dried over anhydrous sodium sulfate and then concentrated to a syrup. The syrup was subjected to deacetylation with ethanolic sodium hydroxide $(53 \%, \mathrm{w} / \mathrm{v})$, treated with Dowex $50\left(\mathrm{H}^{+}\right.$form $)$ and applied to Dowex $1\left(\mathrm{CH}_{3} \mathrm{COO}^{-}\right.$form $)$for separation into acidic oligosaccharide and neutral oligosaccharide.

Smith degradation. ${ }^{14)}$ Samples of polysaccharide ( $100 \mathrm{mg}$, each) were oxidized with $0.03 \mathrm{~m}$ sodium periodate $(100 \mathrm{ml})$ for 14 days at $5^{\circ} \mathrm{C}$ in the dark. After complete oxidation, the oxidized polysaccharide was reduced with sodium borohydride in the usual manner, and a portion of the resulting polysaccharide-polyalcohol was hydrolyzed with $3.5 \mathrm{~N}$ sulfuric acid at $100^{\circ} \mathrm{C}$ for $1 \mathrm{hr}$. The hydrolyzate was neutralized $\left(\mathrm{BaCO}_{3}\right)$, filtered, and the sugar and sugar alcohol were analyzed by GLC on a column with $3 \%$ ECNSS-M on Gas Chrom Q, at $100 \sim 190^{\circ} \mathrm{C}$, programed at a rate of $5^{\circ} \mathrm{C}$ per min, after borohydride reduction followed by acetylation. In another experiment (controlled
Smith degradation), the polysaccharide-polyalcohol was subjected to mild acid hydrolysis with $0.1 \mathrm{~N}$ sulfuric acid at $25^{\circ} \mathrm{C}$ for $20 \mathrm{hr}$. Oligosaccharides which were not degraded by periodate oxidation were fractionated by Bio-gel P-2 column chromatography, and their structures were examined by methylation analysis.

\section{RESULTS AND DISCUSSION}

\section{Methylation analysis}

It was reported in the previous paper that the acidic polysaccharide was composed of galactose, mannose and glucuronic acid in the molar ratio of $\left.5: 4: 2 .{ }^{5}\right)$ But the composition of the acidic polysaccharide was reinvestigated,

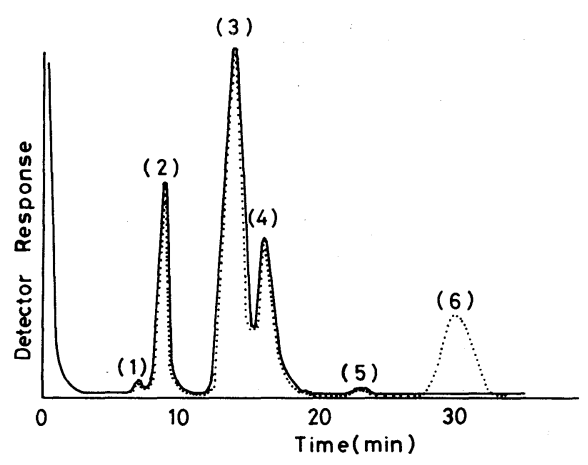

FIG. 1. GLC Profile of the Hydrolysis Products of the Methylated Polysaccharide Produced from Lactose by Strain No. 626 of Aeromonas hydrophila.

Methylated sugars were separated as alditol acetates, on a column of $3 \%$ ECNSS-M on Gas Chrom Q, $180^{\circ} \mathrm{C}$.

- , before reduction; ----, after reduction.

(1) 2,3,4,6-tetra- $O$-methyl-D-mannose; (2) 2,3,4,6-tetra- $O$ methyl-D-galactose; (3) 3,4,6-tri- $O$-methyl-D-mannose; (4) 2,4,6-tri- $O$-methyl-D-galactose; (5) 4,6-di- $O$-methyl-Dmannose; (6) 4,6-di- $O$-methyl-D-glucose.

Table I. Identities and Molar Ratios of Methylated Sugar

Components of the Polysaccharide Produced from Lactose

\begin{tabular}{lll}
\hline \multicolumn{1}{c}{$O$-Methyl sugar } & \multicolumn{1}{c}{$\begin{array}{c}\text { Linkage } \\
\text { indicated }\end{array}$} & $\begin{array}{c}\text { Molar } \\
\text { ratio }\end{array}$ \\
\hline 2,3,4,6-Tetra- $O$-methyl-D-mannose & (Man) $1 \rightarrow$ & 0.06 \\
2,3,4,6-Tetra- $O$-methyl-D-galactose & (Gal) $1 \rightarrow$ & 1.00 \\
3,4,6-Tri- $O$-methyl-D-mannose & $\rightarrow 2$ (Man) $1 \rightarrow$ & 2.00 \\
2,4,6-Tri- $O$-methyl-D-galactose & $\rightarrow 3$ (Gal) $1 \rightarrow$ & 1.30 \\
4,6-Di- $O$-methyl-D-mannose & $\rightarrow 2$ (Man) $1 \rightarrow$ & 0.07 \\
& $\rightarrow 3$ & 1.00 \\
\hline
\end{tabular}


and the molar ratio of galactose, mannose and glucuronic acid was found to be $2: 2: 1$. The acidic polysaccharide $\left([\alpha]_{\mathrm{D}},+108^{\circ} ; \mathrm{MW}, 1.7 \times\right.$ $10^{6}$ ) was methylated by the method of Hakomori, and the methylated sugar fragments formed by acid hydrolysis were examined by GLC, and identified by comparing retention times with standards. As the methylated uronic acid is not detected as the acetyl derivative after borohydride reduction on a column of $3 \%$ ECNSS-M on Gas Chrom Q, the acidic polysaccharide was reduced to the netural polysaccharide. Figure 1 shows GLC separation of the methylated sugars from methylated native and reduced polysaccharide. The evidence of linking of glucose units of the reduced polysaccharide through $\mathrm{C}-1, \mathrm{C}-2$ and $\mathrm{C}-3$ indicates that glucuronic acid in the native polysaccharide was linked through C-1, C-2 and $\mathrm{C}-3$. Therefore, the methylated native polysaccharide seems to consist of $2,3,4,6$ tetra- $O$-methyl-D-mannose, 2,3,4,6-tetra- $O$ methyl-D-galactose, 3,4,6-tri- $O$-methyl-Dmannose, 2,4,6-tri- $O$-methyl-D-galactose, 4,6di- $O$-methyl-D-mannose and 4-mono- $O$ methyl-D-glucuronic acid. In Table I, identities and molar ratios of the methylated sugar components of the acidic polysaccharide are shown. The acidic polysaccharide seems to consist mainly of terminal D-galactose, (1-2)linked-D-mannose, (1-3)-linked-D-galactose and (1-2) and (1-3)-linked-D-glucuronic acid and the branching might occur at C-3 or C-2 of glucuronic acid, to form side chains terminated with galactose residues.

\section{Acetolysis of acidic polysaccharide}

In order to determine the sequences of mannose and galactose, acetolysis of the acidic polysaccharide was carried out and the structures of neutral products were examined. The neutral acetolyzate was separated into mono-, di- and trisaccharide by gel filtration. Paper chromatography of the disaccharide gave two spots, therefore, the disaccharide seemed to be a mixture. Then the mixture was methylated and methylated sugars formed by acid hydrolysis were analyzed by GLC. As shown in

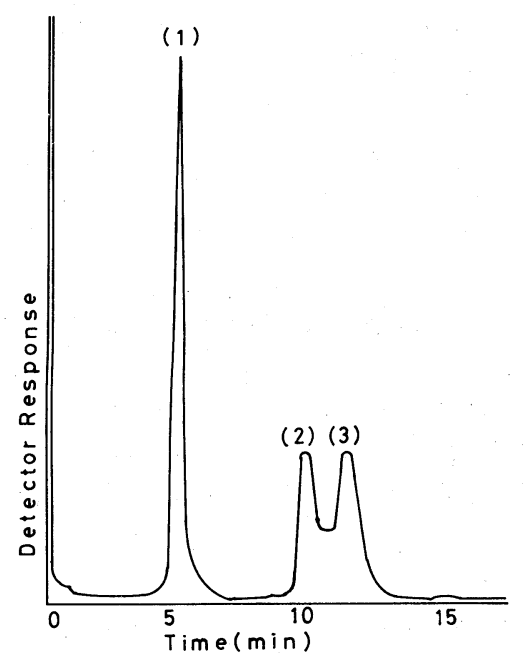

FIG. 2. GLC Profile of the Hydrolysis Products of the Methylated Disaccharide from the Neutral Acetolyzate.

Conditions are same as in Fig. 1.

(1) 2,3,4,6-tetra- $O$-methyl-D-mannose; (2) 3,4,6-tri- $O$ methyl-D-mannose; (3) 2,4,6-tri- $O$-methyl-D-galactose.

Fig. 2, 2, 3, 4, 6-tetra- $O$-methyl-D-mannose, 3,4,6-tri- $O$-methyl-D-mannose and 2,4,6-tri- $O$ methyl-D-galactose were revealed in the ratio of $2: 1: 1$. These disaccharides seemed to have the $\alpha$-cofiguration from specific optical rotation analysis $\left([\alpha]_{D}+68^{\circ}\right.$, in water). From these results, the disaccharides were found to be $\alpha$-mannopyranosyl-(1-2)-D-mannose and $\alpha$-D-mannopyranosyl-(1-3)-D-galactose. $\quad \mathrm{Pa}$ per chromatography of the trisaccharide gave one spot $\left(R_{\mathrm{GLC}}=0.3\right)$, therefore, this trisaccharide seemed to be homogeneous. The hydrolysis products of the methylated trisaccharide gave 2,3,4,6-tetra- $O$-methyl-Dmannose, 3,4,6-tri- $O$-methyl-D-mannose and 2,4,6-tri- $O$-methyl-D-galactose in an equimolar ratio. Ten $\mathrm{mg}$ of the trisaccharide was dissolved in $0.05 \mathrm{~N}$ phosphate buffer, $\mathrm{pH} 4.5$, and incubated at $40^{\circ} \mathrm{C}$ with $\alpha$-mannosidase $(0.1$ units, Jack bean) for $24 \mathrm{hr}$. $\alpha$-Mannosidase activity was then destroyed by heating the reaction mixture in a boiling water bath for $10 \mathrm{~min}$. The products were analyzed by paper chromatography. It was found that the trisaccharide was cleaved into mannose and $\alpha$ mannopyranosyl-(1-3)-D-galactose. On the basis of these results, the trisaccharide was found 
to be $\alpha$-D-mannopyranosyl-(1-2)- $O$ - $\alpha$-D-mannopyranosyl-(1-3)-D-galactose $\left([\alpha]_{\mathrm{D}}+67^{\circ}\right.$, in water).

\section{Partial acid hydrolysis of acidic polysaccharide}

In order to determine the arrangements of D-glucuronic acid residues in the polysaccharide, the acidic polysaccharide was hydrolyzed with $1 \mathrm{~N}$ sulfuric acid at $100^{\circ} \mathrm{C}$ for $4 \mathrm{hr}$. The hydrolyzate was neturalized with $\mathrm{BaCO}_{3}$ and filtered, and the filtrate was passed through columns of Dowex $50\left(\mathrm{H}^{+}\right.$form $)$and Dowex $1\left(\mathrm{CH}_{3} \mathrm{COO}^{-}\right.$form $)$. An acidic component absorbed on Dowex 1 was eluted with $2.5 \mathrm{~N}$ acetic acid, concentrated and then fractionated on a column of Bio-gel P-2. As shown in Fig. 3, the acidic hydrolyzate was separated into three fractions, that is, peak 1, peak 2 and peak 3. Peak 1 was found to contain glucuronic acid and glucuronolactone by paper chromatography. The acidic hydrolyzates of the peak 2 and 3 fractions and their reduced products were subjected to methylation analysis. From the methylated native acidic oligosaccharide of peak 2, only 3,4,6-tri- $O$-methylD-mannose was detected. From the methylated reduced oligosaccharide of peak 2, two peaks were obtained on GLC, corresponding to 2,3,4,6-tetra- $O$-methyl-D-glucose (derived from glucuronic acid) and 3,4,6-tri- $O$-methylD-mannose, in the ratio of $1.0: 1.3$. From these results, the structure of peak 2 was found to be $\alpha$-D-glucopyranosyluronic acid-(1-2)-D-mannose. In the same way, the structure of peak 3 was investigated. Before reduction, only 3,4,6-tri- $O$-methyl-D-mannose was detected, and after reduction, 2,3,4,6-tetra- $O$ methyl-D-glucose (derived from glucuronic acid) and 3,4,6-tri- $O$-methyl-D-mannose were detected in the ratio of $1.0: 2.2$. Therefore, the structure of peak 3 was found to be $\alpha$-Dglucopyranosyluronic acid-(1-2)- $O-\alpha$-D-mannopyranosyl-(1-2)- D-mannose.

\section{Smith degradation of acidic polysaccharide}

From the results of complete Smith degradation of the reduced polysaccharide, glycerol, galactose and glucose (derived from glucuronic acid) were detected and the molar ratio was determined to be $3.2: 1.0: 0.8$ by GLC. This acidic polysaccharide contains relatively large proportions of (1-3)-glycosidic linkages. Information on the sequential arrangements of some of the sugar residues, particularly the linkages between (1-3)-linked residues and their neighboring sugar residues

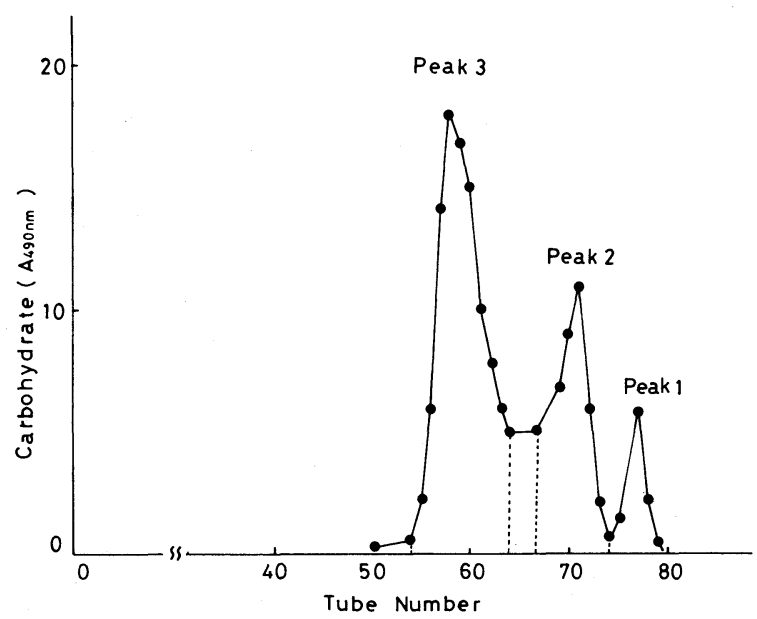

FIG. 3. Gel Filtration Profile of the Partial Acidic Hydrolyzate from the Acidic Polysaccharide.

Partial acidic hydrolysis was performed with $1 \mathrm{~N} \mathrm{H}_{2} \mathrm{SO}_{4}$ for $4 \mathrm{hr}$ at $100^{\circ} \mathrm{C}$. The hydrolyzate was applied to a column of Bio-gel P-2 $(3.5 \times 157 \mathrm{~cm})$ and eluted with water at a flow rate of $18 \mathrm{ml}$ per hr. Fractions of $10 \mathrm{ml}$ were collected, and their carbohydrate content was measured by the phenol-sulfuric acid method. 


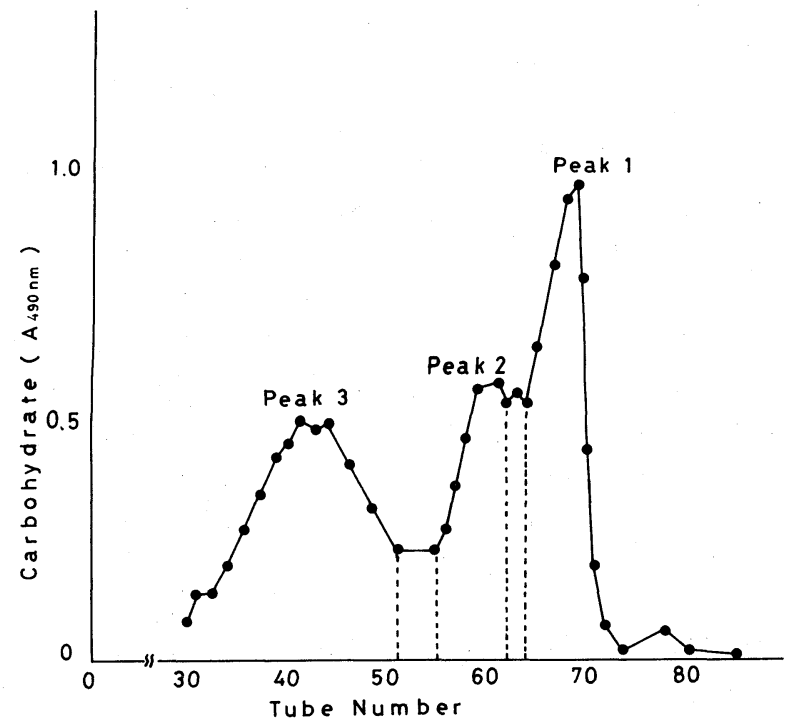

FIG. 4. Gel Filtration of Oligosaccharides Produced by Controlled Smith Degradation of the Acidic Polysaccharide.

Conditions are same as in Fig. 3.

may be obtained by controlled Smith degradation. The acidic polysaccharide oxidized with sodium periodate was reduced with borohydride to the corresponding polyalcohol and controlled Smith degradation $(0.1 \mathrm{~N}$ $\mathrm{H}_{2} \mathrm{SO}_{4}, 20 \mathrm{hr}$ at room temperature) of this glycan polyalcohol was carried out. The hydrolyzate of the glycan polyalcohol was applied to a column of Bio-gel P-2. As shown in Fig. 4, three fractions were obtained, that is, peak 1 , peak 2 and peak 3 . Peak 1 and peak 2 were methylated, and their structures were determined by comparing the peaks of the hydrolysis products of the native and reduced oligosaccharides. GLC revealed that the hydrolysis products of methylated reduced peak 1 gave 2,3,4,6-tetra- $O$-methyl-D-galactose and 2,4,6-tri- $O$-methyl-D-glucose (derived from glucuronic acid) in the ratio of $1.0: 1.3$. From this result, peak 1 may be $\alpha$-D-galactopyranosyl-(1-3)- $O$ - $\alpha$-D-glucopyranosyluronic acid-(1-2)-D-glycerol. In case of peak 2, GLC revealed that the hydrolysis of peak 2 gave 2,3,4,6-tetra- $O$-methyl-D-galactose, 2,4,6-tri$O$-methyl-D-glucose (derived from glucuronic acid) and 2,4,6-tri- $O$-methyl- D-galactose in the ratio of $1.0: 1.3: 1.2$. From this re- sult and the production of aldobiouronic acid ( $\alpha$-D-glucopyranosyluronic acid-(1-2)-Dmannose) by partial acid hydrolysis, peak 2 may have the sequence of $\alpha$-D-galactopyranosyl-(1-3)- $\alpha$-D-galactopyranosyl-(1-3)- $\alpha$-Dglucopyransyluronic acid-(1-2)-D-glycerol. From the results of GLC of methylated peak 3, it seemed to be hydrolyzed incompletely with $0.1 \mathrm{~N}$ sulfuric acid for $20 \mathrm{hr}$. After controlled Smith degradation, 4,6-di- $O$-methyl-D-glucose from the reduced products of Smith degradation disappeared but 2,4,6-tri- $O$-methyl-Dglucose was found. Therefore, one-reducing terminal galactose residue seemed to be attached to the glucuronic acid at the C-2 position. As reported in our previous paper, strain No. 626 of Aeromonas hydrophila was found to produce levan and an acidic polysaccharide from sucrose and raffinose, and mainly an acidic polysaccharide from monosaccharides and disaccharides other than sucrose. The acidic polysaccharide produced from lactose consisted of galactose, mannose and glucuronic acid in the ratio of $2: 2: 1$. On methylation analysis, it was seen that mannose residues were linked at $\mathrm{C}-1$ and $\mathrm{C}-2$ positions and galactose residues at $\mathrm{C}-1$ and $\mathrm{C}-3$ po- 


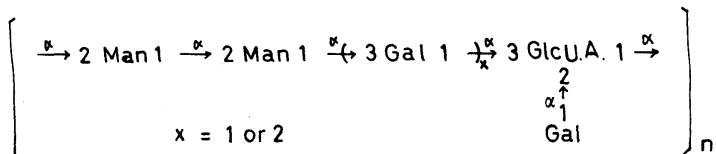

Fig. 5. A Possible Structure for the Acidic Polysaccharide Produced by Strain No. 626 of Aeromonas hydrophila.

sitions and only the $\mathrm{C}-1$ position. Glucuronic acid residues were linked at C-1, C-2 and C-3 positions. After controlled Smith degradation, 4-mono- $O$-methyl-D-glucuronic acid disappeared but 2,4-di- $O$-methyl-D-glucuronic acid was revealed. Therefore, one-reducing terminal galactose residue seemed to be attached to the (1-3)-linked-D-glucuronic acid residue at the $\mathrm{C}-2$ position. The glycosidic linkages suggested by the methylation analysis were supported by the results of complete Smith degradation, which produced glycerol, galactose and glucuronic acid in the ratio of $3.2: 1.0: 0.8$. After acetolysis, two neutral disaccharides and one neutral tri-saccharide were found. The neutral disaccharides were found to be $\alpha$-Dmannopyranosyl-(1-2)-D-mannose and $\alpha$-Dmannopyranosyl-(1-3)-D-galactose. The neutral trisaccharide seemed to be $\alpha$-D-mannopyranosyl-(1-2)- $O-\alpha$-D-mannopyranosyl-(1-3)D-galactose. The partial acid hydrolysis of the acidic polysaccharide with $1 \mathrm{~N}$ sulfuric acid for $4 \mathrm{hr}$ produced aldobiouronic acid and aldotriouronic acid, which were found to be $\alpha$-D-glucopyranosyluronic acid-(1-2)-Dmannose and $\alpha$-D-glucopyranosyluronic acid-(1,2)- $O$ - $\alpha$-D-mannopyranosyl-(1-2)-Dmannose, respectively. As (1-3)-linked sugar residues are not cleaved by periodate oxidation, controlled Smith degradation indicates the detailed structure of (1-3)-linked sugars. (1-3)-linked-D-galactose and (1-3)-linkedD-glucuronic acid were determined to exist in this acidic polysaccharide by methylation and complete Smith degradation analysis, moreover, controlled Smith degradation was carried out. The products of controlled Smith degradation were found to be $\alpha-\mathrm{D}$ galactopyranosyl-(1-3)- $O$ - $\alpha$-D-glucopyranosyluronic acid-(1-2)-D-glycerol and $\alpha$-D-galactopyranoxyl-(1-3)- $O-\alpha-\mathrm{D}-$ galactopyranoxyl(1-3)- $O$ - $\alpha$-D-glucopyranosyluronic acid-(1-2)D-glycerol. On the basis of these results, a possible structure for the acidic polysaccharide was proposed, as shown in Fig. 5.

Acknowledgment. The authors wish to thank Professor Akira Misaki of the Department of Food and Nutrition, Faculty of Science of Living, Osaka City University, for his technical advice.

\section{REFERENCES}

1) G. A. Adams and S. M. Martin, Can. J. Biochem., 42, 1403 (1964).

2) J. F. Wilkinson, W. F. Dudman and G. O. Aspinall, Biochem. J., 59, 446 (1955).

3) T. Harada, Arch. Biochem. Biophys., 112, 37 (1965).

4) G. H. Cohen and D. B. Johnstone, J. Bacteriol., 88, 1695 (1964).

5) S. Ueda, F. Momii, K. Osajima and K. Ito, Agric. Biol. Chem., 45, 1977 (1981).

6) W. E. Trevelyan, D. P. Procter and J. S. Harrison, Nature (London), 166, 444 (1950).

7) A. Misaki, N. Seto and I. Azuma, J. Biochem., 76, 15 (1974).

8) M. Dubois, K. A. Gilles, J. K. Hamilton, P. A. Rebers and F. Smith, Anal. Chem., 28, 350 (1956).

9) M. Somogyi, J. Biol. Chem., 195, 19 (1952).

10) W. F. Dudman, Carbohyd. Res., 66, 9 (1978).

11) R. L. Taylor and H. E. Conrad, Biochemistry, 11, 1383 (1972).

12) A. Sandford and H. E. Conrad, Biochemistry, 5, 1508 (1966).

13) S. Hakomori, J. Biochem., 55, 205 (1964).

14) I. J. Goldstein, G. W. Hay, B. A. Levis and F. Smith, "Methods in Carbohydrate Chemistry," Vol. 5, 1965 , p. 361 . 